\title{
28 Research Soure \\ Efficacy and safety of oral zolmitriptan in the preictal phase of episodic cluster headache
}

Wei Liu

First Affiliated Hospital of Soochow University https://orcid.org/0000-0002-9155-1179

Jiajie Lu

First Affiliated Hospital of Soochow University

\section{Xiaoning Guo}

First Affiliated Hospital of Soochow University

Chunyang Xu

Traditional Chinese Medicine Hospital of Kunshan

\section{Manyun Yan}

Changshu NO 2 Peoples' Hospital

\section{Quanquan Zhang}

First Affiliated Hospital of Soochow University

Jianqiang $\mathrm{Ni}$

First Affiliated Hospital of Soochow University

Haifeng Lu

First Affiliated Hospital of Soochow University

Hongru Zhao ( $\nabla$ tiantan11@163.com )

The First Affiliated Hospital of Soochow University

\section{Research article}

Keywords: episodic cluster headache, phases, preictal phase, zolmitriptan, efficacy

Posted Date: June 18th, 2021

DOI: https://doi.org/10.21203/rs.3.rs-629591/v1

License: (c) (1) This work is licensed under a Creative Commons Attribution 4.0 International License.

Read Full License 


\section{Abstract}

\section{Background}

Recent studies on cluster headache suggested that headache includes three phases.Oral zolmitriptan is effective in the treatment of acute headache attack. We aim to describe the clinical characteristics of different phases of episodic cluster headache $(\mathrm{eCH})$ and observe the efficacy and safety of oral zolmitriptan in preictal phase of eCH attacks.

\section{Methods}

Forty-eight patients with $\mathrm{eCH}$ were enrolled in this study. We divided the $\mathrm{CH}$ attack into three phases: preictal, ictal and postictal phase and analyzed the clinical features of each phase. We collected the data of twenty-three patients who took zolmitriptan in the preictal phase of three consecutive $\mathrm{CH}$ attacks. The Visual Analogue Scale (VAS) score and duration of headache after three consecutive treatments were compared with the baseline respectively. Chi square test, $t$ test, Mann Whitney $U$ test, Pearson linear correlation analysis, paired t-test and paired non-parametric rank-sum test were used for statistical analysis.

\section{Results}

Forty-five patients (93.8\%) reported the presence of preictal symptoms. The most frequent preictal symptom was mild to moderate head discomfort in the area of the subsequent serious headache, accounting for $87.5 \%$. The duration of preictal period was positively correlated with the duration of headache. The Pearson correlation coefficient was $0.313(P=0.018)$. Among the twenty-three patients taking oral zolmitriptan in the preictal phase, the VAS max score was significantly lower than that of baseline headache $(P<0.01)$. The duration of headache after three preictal administration was significantly shorter than that of baseline headache $(P<0.01)$. The incidence of adverse reactions after taking zolmitriptan was $10.1 \%$, including head drowsiness, neck stiffness and mild chest tightness.

\section{Conclusions}

Preictal symptoms were common in $\mathrm{CH}$. Mild to moderate discomfort in the subsequent serious headache was the most common symptom in the preictal phase. Oral administration of zolmitriptan in the preictal period could reduce the severity of headache and shorten the duration of headache in a cluster period without serious adverse reactions.

\section{Background}

Cluster headache is a trigeminal autonomic cephalalgia which is characterized by strictly unilateral headache attacks lasting 15-180 minutes and occurring up to eight times a day accompanied by at least one autonomic symptom ipsilateral to the pain or a sense of agitation, or both[1]. It can be divided into episodic cluster headache $(\mathrm{eCH})$ and chronic cluster headache $(\mathrm{cCH})$. $\mathrm{ECH}$ accounts for $90 \%$ of $\mathrm{CH}[2]$. 
Patients with eCH have at least two cluster periods lasting for 7 days to 1 year without treatment, and the remission period is $\geq 1$ month. Owing to the excruciating and highly-frequent recurrence during the cluster bout, $\mathrm{CH}$ has been referred to as the "suicide headache". 15.9\% of Asian patients suffering from acute headache attack had suicidal ideation, while the suicidal ideation decreased significantly during headache intermission[3]. The recurrent intolerable attacks of the disorder seriously affected the patient's work and life[4] and caused heavy social and economic burden[5]. Recent studies have found that one attack of $\mathrm{CH}$ did not just include pain phase. Preictal symptoms were very frequent in $\mathrm{CH}[6]$. Given that these symptoms occur relatively early in the preictal phase and are frequent, there could be a potential benefit if starting abortive treatment when these symptoms occur. While nowadays the treatment of acute attack has been mainly conducted in the ictal phase, in which the attack had already made a serious impact on patient. Studies found that the clinical benefits were more pronounced when the patients took medicine at the initial period of migraine attack than delayed medication[7-8]. The pathophysiology of $\mathrm{CH}$ was similar to that of migraine to some extent, so we speculated that administration in preictal phase of $\mathrm{CH}$ could effectively relieve or terminate the headache attack as soon as possible. We aimed to investigate the clinical characteristics of the preictal phase of $\mathrm{eCH}$ in our headache clinic and to observe the efficacy and safety of oral zolmitriptan in the preictal phase of eCH patients within the cluster bout.

\section{Methods}

\section{Study design and Participants}

According to ICHD-3 (Table 1), patients diagnosed with eCH in an active disease state were invited to participate in the study. A total of 48 patients diagnosed with $\mathrm{eCH}$ in the outpatient department of Neurology and headache clinic of the First Affiliated Hospital of Soochow University were recruited from October 2018 to December 2020. We collected detailed information through a structured questionnaire survey. Our study was approved by the medical ethics committee of The First Affiliated Hospital of Suzhou University (approval No: 2020; ethics committee file name No. 162). Informed consent was obtained by the patient and family members.

The inclusion criteria were as follows: diagnosed as eCH; aged from 18 years to 65 years old; within the cluster bout. Exclusion criteria were as follows: combined with other primary or secondary headache; head trauma; abnormal findings of CT or MRI of head; drug or alcohol abuse; intracranial infection and other serious systemic diseases; cardiovascular disease or severe hypertension; allergic to triptan; preventive treatment of $\mathrm{CH}$ and pregnancy.

\section{Table 1 Diagnostic criteria for cluster headache[1]}


Diagnostic criteria for cluster headache

A. At least five attacks fulfilling criteria B-D

B. Severe or very severe unilateral orbital, supraorbital and/or temporal pain lasting 15-180 minutes (when untreated)

C. Either or both of the following:

1. at least one of the following symptoms or signs, ipsilateral to the headache:

a) conjunctival injection and/or lacrimation

b) nasal congestion and/or rhinorrhoea

c) eyelid oedema

d) forehead and facial sweating

e) miosis and/or ptosis

2. a sense of restlessness or agitation

D.Occurring with a frequency between one every other day and eight per day

E. Not better accounted for by another ICHD-3 diagnosis

\section{Clinical data collection}

All patients completed a structured questionnaire designed for the assessment of eCH. We collected clinical information about current and previous bouts, including age, gender, family history of headache, course of disease, location, manifestation of headache (tabbing pain, dull sensation, distending pain and throbbing pain), headache duration, frequency, severity (VAS score, numeric rating scale from 0 to 10: 0 meant painless; 1-3 mild pain; 4-6 moderate pain; 7-8 severe pain; 9-10 extremely severe pain)[9], diurnal rhythm, inducements, phases and accompanying symptoms.

We collected the clinical data of 23 patients who took zolmitriptan during three consecutive preictal phases, including the baseline clinical data, medication time, VAS scores at 15 minutes, 30 minutes and 60 minutes after treatment, VAS max score, duration of headache and adverse reactions. The VAS score and duration after three consecutive medications were compared with that of the baseline, respectively.

Zolmitriptan was produced by wante Pharmaceutical (Hainan) Co., Ltd., and the trade name is zolmitriptan tablets (kaqu), national drug approval word H20061077. The dose was $2.5 \mathrm{mg} /$ tablet. The administration in preictal phase defined as oral administration of zolmitriptan within 15 minutes of preictal phase[8].

\section{Phases and accompanying symptoms}

We divided the phases into three sections: preictal phase, ictal phase and postictal phase. The ictal phase was defined according to the ICHD-3 as the period of severe or extremely severe periorbital and/or 
temporal pain with accompanying symptoms or restlessness. The preictal phase was defined as the time that pain symptoms, autonomic nerve symptoms or other symptoms began to appear until severe or very serious pain occurred. The postictal phase was the time that severe pain got into mild until it disappeared and other accompanied symptoms disappeared completely.

All symptoms were grouped into 3 categories: local and painful symptoms (comprising local painful sensations in the subsequent severe headache, fullness of the ear, neck pain); local and painless symptoms and signs (conjunctival injection, lacrimation, rhinorrhea, nasal congestion, forehead『facial sweating $\bowtie$ ptosis or miosis) and general symptoms (difficulty concentrating, restlessness, mood changes, etc).

\section{Data comparison}

The $\mathrm{eCH}$ patients with preictal phase were divided into two groups, according to the duration of preictal phase, $\leq 15 \mathrm{~min}$ group and $>15 \mathrm{~min}$ group. The number of symptoms, headache severity in the ictal phase and headache duration were compared between the two groups. For $23 \mathrm{eCH}$ patients treated within preictal phase, the VAS score and duration after three-time treatments were compared with that of the baseline respectively.

\section{Statistical analyses}

Statistical analyses were performed using SPSS23.0 software. Chi square test was used for categorical variables, which were presented with the number of cases (\%). T-test was used for continuous variables in accordance with normal distribution, which were presented with mean \pm standard deviation. The metrological data that did not conform to the normal distribution were represented by the median (quartile range), using Mann-Whitney $U$ test. Pearson linear correlation analysis was used for correlation analysis. $P<0.05$ was statistically significant.

The VAS score before and after treatment was presented with medians and interquartile ranges (IQR). The duration was presented with mean and standard deviation. The duration of headache after treatments in the preictal phase for three times was respectively compared with that at baseline by paired t-test, and VAS scores by paired non-parametric rank-sum test, and the test level was adjusted to $0.017(0.05 / 3)$.

\section{Results}

\section{Demographic and clinical characteristics of $\mathrm{eCH}$}

A total of $48 \mathrm{eCH}$ patients were recruited in the study. The male to female ratio was $5: 1$. The median age of patients was $33 \pm 8.1$ years old and median disease duration was $8.2 \pm 6.2$ years. The demographic and clinical characteristics of enrolled patients were listed in Table 2. Forty-six patients (95.8\%) had unilateral headache and only 2 patients $(4.2 \%)$ had bilateral headache. Most of the patients developed pain in multiple locations: orbital $62.5 \%$, temporal $60.4 \%$, supraorbital $52.1 \%$, frontal $43.8 \%$, occipital $27.1 \%$, parietal region $14.6 \%$ and neck $4.2 \%$ (Figure 1 ). 
Table 2 Demographic and clinical characteristics of 48 patients with episodic cluster headache [Case (\%)] 


\begin{tabular}{|c|c|}
\hline Demographic and clinical features & Statistic \\
\hline Male/Female & $40(83.3) / 8(16.7)$ \\
\hline Age (Year $₫ \mathbf{s})$ & $33 \pm 8.1$ \\
\hline Course of Disease (Years $₫ \pm s$ ) & $8.2 \pm 6.2$ \\
\hline \multicolumn{2}{|l|}{ Frequency of headache } \\
\hline Every other day & 16(33.3) \\
\hline Once per day & $40(83.3)$ \\
\hline Twice per day & 19(39.6) \\
\hline More than three times a day & $5(10.4)$ \\
\hline 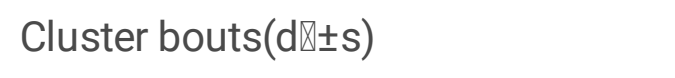 & $29.7 \pm 16.8$ \\
\hline \multicolumn{2}{|l|}{ Circadian } \\
\hline Day & $31(64.6)$ \\
\hline Night & $4(8.3)$ \\
\hline Day and night & 13(27.1) \\
\hline Family history & $7(14.6)$ \\
\hline VAS max & $9 \llbracket 8,10 \rrbracket$ \\
\hline Headache duration (min $₫ \pm s)$ & $81.8 \pm 36.9$ \\
\hline \multicolumn{2}{|l|}{ Inducements } \\
\hline Smoking & $2(4.2)$ \\
\hline Drinking & $12(25.0)$ \\
\hline Climate change & 16(33.3) \\
\hline Cool wind & $5(10.4)$ \\
\hline Sleep disorders & $17(35.4)$ \\
\hline Mood changes & $4(8.3)$ \\
\hline Mental stress & $11(22.9)$ \\
\hline Special smell & $5(10.4)$ \\
\hline Fatigue & $3(6.3)$ \\
\hline \multicolumn{2}{|l|}{ Psychiatric comorbidities } \\
\hline Anxiety & $21 \rrbracket 43.8 \rrbracket$ \\
\hline
\end{tabular}




\section{Clinical characteristics of the preictal phase of eCH}

Among the $48 \mathrm{eCH}$ patients included in our study, 45 patients (93.8\%) reported the presence of preictal symptoms. The most frequent preictal symptom was mild to moderate head discomfort in the area of the subsequent attack, accounting for $87.5 \%$. Forehead and facial sweating was reported in 9 patients (18.7\%), lacrimation in $7(14.5 \%)$ and nasal congestion in $6(12.5 \%)$ during the preictal phase. The frequent general symptoms were mood changes (33.3\%), difficulty in concentration (31.2\%) and restlessness (31.2\%).

\section{Group comparisons}

Among the $48 \mathrm{eCH}$ patients, 45 had preictal phase while 3 patients reported no obvious preictal phase. The preictal phase duration ranged from 5 to $60 \mathrm{~min}$, of which 5-30 min was the most common (71\%). (Figure 2). The duration of headache in the group with preictal phase $>15 \mathrm{~min}$ was longer than that $\leq 15$ min, and the difference between two groups was statistically significant $(P=0.018)$ (Table 3$)$. The duration of preictal phase was positively correlated with headache duration (Pearson correlation coefficient was 0.313 ). There was no correlation on the number of symptoms and VAS score between the two groups.

Table 3 Analysis of related factors by grouping the duration of preictal period

\begin{tabular}{|llll|}
\hline Group & $\begin{array}{l}\text { Number of symptoms in the Ictal } \\
\text { phase }\end{array}$ & VAS & $\begin{array}{l}\text { Headache duration } \\
\text { (min) }\end{array}$ \\
\hline $\begin{array}{l}\text { Preictal phase } \leq 15 \mathrm{~min} \\
\text { group }\end{array}$ & $9(6.8,14)$ & $9(8,10)$ & $74.3 \pm 36.6$ \\
\hline $\begin{array}{l}\text { Preictal phase }>15 \mathrm{~min} \\
\text { group }\end{array}$ & $13(8,15)$ & $9(8,10)$ & $102 \pm 32.7$ \\
\hline P value & 0.114 & 0.373 & $0.018^{\star}$ \\
\hline
\end{tabular}

T-test was used for continuous variables in accordance with normal distribution, which were presented with mean \pm standard deviation. The metrological data that did not conform to the normal distribution were represented by the median (quartile range), using Mann-Whitney U test. ${ }^{*} \mathrm{p}<0.05$.

\section{Efficacy of oral zolmitriptan in the preictal phase for three consecutive times}

The median score of VAS max after three medications were all 5 , while the baseline was 9 . The duration of attack after three times administration of zolmitriptan was $48.2 \pm 24.5$ minutes, $45.7 \pm 21.8$ minutes, 
$52.6 \pm 31.9$ minutes, respectively, while the baseline was 75.7 \pm 38.1 minutes. The differences in VAS max scores and duration before and after treatment were statistically significant $(P<0.01)($ Table 4).

\section{Table 4 Comparisons between baseline and post-treatment}

\begin{tabular}{|c|c|c|c|c|}
\hline & Baseline & First treatment & Second treatment & Third treatment \\
\hline VAS max & $9 \otimes 8,9 \rrbracket$ & $5 \rrbracket 4,7 \nabla^{\star}$ & $583,7 \bigotimes^{*}$ & $5 \otimes 3,7 \nabla^{*}$ \\
\hline Duration/min & $75.7 \pm 38.1$ & $48.2 \pm 24.5^{\star}$ & $45.7 \pm 21.8^{*}$ & $52.6 \pm 31.9 *$ \\
\hline
\end{tabular}

The duration of headache after treatments in the preictal phase for three times was respectively compared with that at baseline by paired t-test, and VAS scores by paired non-parametric rank-sum test, and the test level was adjusted to $0.017(0.05 / 3) .{ }^{*} p<0.01 \rrbracket a=0.017$

The proportions of mild or painless headache at 30 minutes after each treatment were $56 \%, 70 \%$ and $61 \%$, respectively. No difference was found among the three groups. After three-time preictal administration, the median VAS scores were 3,3,2, respectively. Scores at 15 minutes were $5,4,4,30$ minutes were 2,1,1 and 60 minutes were all 0 (Figure 3).

\section{Adverse reactions}

For the first treatment, two patients felt dizzy and one patient reported dizziness and mild chest tightness. One patient was accompanied with drowsiness and two patients with dizziness and neck stiffness for twice. The incidence of adverse reactions in our study was $10.1 \%$, including dizziness, neck stiffness, drowsiness and mild chest tightness, all of which were relatively mild and could be relieved spontaneously.

\section{Discussion}

We conducted a single-center observational study to investigate the clinical characteristics of preictal phase of eCH and evaluate the efficacy and safety of oral zolmitriptan in the preictal phase. Our main findings were as follows: preictal symptoms were observed in most of the eCH patients, and the preictal duration was positively correlated with the headache duration within the current bouts. Preictal administration of zolmitriptan in $\mathrm{eCH}$ patients could significantly shorten headache duration, reduce the headache severity, and prevent progression of severe headache.

In our study, preictal symptoms occurred in forty-five (93.8\%) patients. The duration of preictal phase was less than 1 hour, usually lasting for 5 to 30 minutes, which was in line with the study in Korean[10] and Rossi study[11], significantly shorter than the duration of migraine preictal symptoms. Mild to moderate discomfort in the subsequent serious headache was the most common preictal symptom, accounting for $87.5 \%$, manifesting as dull, stabbing and distending sensation. Blau[12] and Engel[13] were the first to investigate pre-attack symptoms of $\mathrm{CH}$. They defined the local and painful symptoms as "pain 
accumulation" of the headache. The occurrence of neck pain in preictal phase accounted for $4.1 \%$, which indicated that the disfunction of trigeminal cervical complex and hypothalamus may be involved in the pathophysiology of $\mathrm{CH}$ preictal phase. Among the autonomic symptoms, conjunctival congestion and lacrimation were observed in $10 \%$ of $\mathrm{CH}$ patients, and the incidence of those symptoms was significantly lower than the $40 \%$ reported in the Western countries[13], similar to the incidence of $11 \%$ reported by Soohyun Cho in Korea[10]. None of the patients in this study reported miosis in the preictal phase, probably because this is difficult to determine if not paid specific attention.

Mood changes and difficulty in concentration occurred in $33.3 \%$ and $31.2 \%$ of our patients, respectively. Previous neuroimaging research showed that the frontal cortex may be involved in the pathophysiology of the preictal phase. MR diffusion tensor imaging study demonstrated that the white matter connection of different hypothalamic nuclei showed increasing trend of connection between right frontal and cerebral hemisphere[14]. Changes in mood and attention suggested the involvement of limbic system. While yawning and physical energy decrease may be mediated by the central dopaminergic system[1516].In the study of Laurell, about one third of migraine patients had preictal symptoms such as yawning, mood changes, restlessness and photophobia, which directly or indirectly indicated that the hypothalamus was involved in the pathophysiology of migraine[17].The recent neuroimaging studies of migraine have confirmed that hypothalamic activation was involved in the occurrence of migraine preictal symptoms. Alterations in the functional connections between the hypothalamus, the trigeminal nucleus and the rostral pons promoted the procession of migraine[18-21]. It was well known that hypothalamus and trigeminal nerve system were involved in the pathophysiological mechanism of $\mathrm{CH}$, therefore the preictal symptoms may be related to the activation of these sites[20]. Previous studies showed that the trigeminal neurovascular system, parasympathetic fibers, and hypothalamus took part in the onset of $\mathrm{CH}$ attack. Soohyun Cho speculated that these above structures may be involved in the early phase before the onset of $\mathrm{CH}[10]$. The most recent study of $\mathrm{CH}$ proposed the theory of "shadow attacks", which referred to the low-level self-limiting ipsilateral pain that occurred preceding the first episodic attack in the upcoming $\mathrm{CH}$ bout[22-23]. The investigators considered the symptoms as predictive sign for cluster bout[24]. Further dynamic functional imaging studies on the "shadow attacks" could be helpful to reveal the pathophysiology of the disease.

In our study, we found that the duration of preictal phase was positively correlated with the headache duration (Pearson's correlation coefficient was 0.313 ) while the numbers of symptoms and the severity of headache had no correlation with the duration of preictal phase. Combined with the previous studies of $\mathrm{CH}$, it was known that the plasma concentrations of Calcitonin gene-related peptide (CGRP) and Vasoactive intestinal peptide (VIP) increased during attacks[25]. Studied on experimental animal models demonstrated that trigeminal nerve stimulation could lead to the release of CGRP, VIP, Pituitary adenylate cyclase activating polypeptide (PACAP) and other inflammatory peptides. PACAP could induce the activation of related neurons (such as superior salivatory nucleus(SSN), sphenopalatine ganglion(SPG) and trigeminal ganglion (TG), and drive the onset of typical symptoms. This could lead to the speculation that preictal administration may block or reduce the release of inflammatory peptides and the activation of related neurons, thus shorten the duration of headache and decrease the severity of headache. 
Zolmitriptan is a selective agonist of 5-hydroxytryptamine 1B/1D receptor subtype[26], which is effective and safe for acute treatment of migraine and cluster headache. Nasal zolmitriptan is generally regarded as the acute therapy of $\mathrm{CH}$ with a level A recommendation by American Headache Society (AHS) guidelines, and oral zolmitriptan with a level B recommendation. The elimination half-life of zolmitriptan and its metabolites was about 3 hours[27]. The intervals of the drug administration of our patients were longer than the maintenance time and three half-lives of zolmitriptan, so the cumulative effect of drugs on the next treatment can be ruled out. As seen in previous $\mathrm{CH}$ studies, $57 \%$ of $\mathrm{CH}$ patients felt mild pain or painless after taking zolmitriptan $5 \mathrm{mg}$ for 30 minutes[28], while the rates in our study were $56-70 \%$ after taking zolmitriptan $2.5 \mathrm{mg}$ for 30 minutes. This may imply that preictal treatment of eCH could achieve the same or even better curative effect with a smaller dose zolmitriptan.

The possible reasons for the effective treatment of $\mathrm{CH}$ in the preictal phase were probably because that zolmitriptan was shown to be inhibit timely the release of vasoactive peptides via central and peripheral 5-HT1B receptors, and thus stop the cascade of neurogenic inflammation earlier. It regulated the intracellular calcium promptly so as to block the synaptic transmission between the terminals and body of peripheral trigeminal nerve neurons, as well as inhibited the pain information transmission mediated by CGRP, substance $\mathrm{P}(\mathrm{SP})$ and glutamate. Moreover, zolmitriptan played a therapeutic role by constricting the blood vessels and inhibiting the dilation of blood vessels. Besides, we could not ignore the presence of nausea. In this study, $10.4 \%$ of the patients reported nausea in the preictal stage and $60 \%$ in the ictal phase. The symptoms of nausea and vomiting in migraine patients led to delayed gastric emptying, which affected the absorption of oral drugs[29]. We speculated that the drug absorption in the preictal phase may be more complete than that in ictal phase.

There were some limitations in our study. Firstly, it was a single center observational study with a small sample size. The incidence rate of disease was lower than that in European and American countries and the research cycle was limited. In addition, due to ethical limitations, we could not compare the efficacy of prodromal and delayed administration. Further randomized, placebo-controlled studies are needed to assess the exact therapeutic effect in the preictal phase, and the results will be more convincing.

\section{Conclusion}

Our study demonstrated that preictal phase was frequent in $\mathrm{eCH}$ patients and preictal duration was positively correlated with the headache duration. After oral administration of zolmitriptan in the preictal phase, the severity of headache was lower and the duration of headache was shorter than that at baseline, and the therapeutic effect was consistent in a cluster period without any serious adverse reactions. Since the origin of $\mathrm{CH}$ attacks is unresolved, our study of preictal symptoms and early abortive treatment strategies could contribute to the understanding of $\mathrm{CH}$ pathophysiology.

\section{Abbreviations}


eCH: episodic cluster headache; $\mathrm{CCH}$ :chronic cluster headache;ICHD-3:International Classification of Headache Disorders,3rd edition;VAS:visual analogue scale;SSN:superior salivatory nucleus;SPG:sphenopalatine ganglion;CGRP:Calcitonin gene-related peptide;VIP:Vasoactive intestinal peptide;PACAP:Pituitary adenylate cyclase activating polypeptide;TG:trigeminal ganglion;SP:substance P;

\section{Declarations}

\section{Acknowledgements}

The authors thank professor Hongru Zhao for proofreading the paper.

\section{Authors' contributions}

All authors designed the review and drafted the manuscript. All authors read and approved the final manuscript.

\section{Funding}

Not applicable.

\section{Availability of data and materials}

Not applicable.

\section{Ethics approval and consent to participate}

The First Affiliated Hospital of Suzhou University approved the study,and all participants provided written informed consent.

\section{Consent for publication}

Not applicable.

\section{Competing interests}

Not applicable.

\section{References}

1. Headache Classification Committee of the International Headache Society (IHS) The International Classification of Headache Disorders, 3rd edition. Cephalalgia. Jan 2018;38(1):1-211

2. Giani L, Proietti Cecchini A, Leone M (2020) Cluster headache and risk of chronic transformation. Neurol Sci Dec 41(Suppl 2):497-498 
3. Ji Lee M, Cho SJ, Wook Park J et al (2019) Increased suicidality in patients with cluster headache. Cephalalgia Sep 39(10):1249-1256

4. Rozen TD, Fishman RS (2012) Cluster headache in the United States of America: demographics, clinical characteristics, triggers, suicidality, and personal burden. Headache Jan 52(1):99-113

5. D'Amico D, Raggi A, Grazzi L, Lambru G. Disability (2020) Quality of Life, and Socioeconomic Burden of Cluster Headache: A Critical Review of Current Evidence and Future Perspectives. Headache Apr 60(4):809-818

6. Snoer A, Lund N, Beske R, Hagedorn A, Jensen RH, Barloese M (2018) Cluster headache beyond the pain phase: A prospective study of 500 attacks. Neurology Aug 28(9):e822-e831 91(

7. Goadsby PJ, Massiou H, Pascual J et al (2007) Almotriptan and zolmitriptan in the acute treatment of migraine. Acta Neurol Scand Jan 115(1):34-40

8. Klapper J, Lucas C, Rosjo O, Charlesworth B, group Zs (2004) Benefits of treating highly disabled migraine patients with zolmitriptan while pain is mild. Cephalalgia Nov 24(11):918-924

9. Haefeli M, Elfering A (2006) Pain assessment. Eur Spine J Jan 15(Suppl 1):S17-S24

10. Cho S, Cho SJ, Lee MJ et al (2021) Clinical characteristics of pre-attack symptoms in cluster headache: A large series of Korean patients. Cephalalgia Feb 41(2):227-236

11. Rossi P, Ambrosini A, Buzzi MG (2005) Prodromes and predictors of migraine attack. Funct Neurol Oct-Dec 20(4):185-191

12. Blau JN, Engel HO (1998) Premonitory and prodromal symptoms in cluster headache. Cephalalgia Mar 18(2):91-93; discussion 71-92

13. Torelli P, Manzoni GC (2003) Pain and behaviour in cluster headache. A prospective study and review of the literature. Funct Neurol Oct-Dec 18(4):205-210

14. Lemaire JJ, Frew AJ, McArthur D et al (2011) White matter connectivity of human hypothalamus. Brain Res Jan 31:1371:43-64

15. Akerman S, Goadsby PJ (2007) Dopamine and migraine: biology and clinical implications. Cephalalgia Nov 27(11):1308-1314

16. Charbit AR, Akerman S, Goadsby PJ (2010) Dopamine: what's new in migraine? Curr Opin Neurol Jun 23(3):275-281

17. Maniyar FH, Sprenger T, Monteith T, Schankin CJ, Goadsby PJ (2015) The premonitory phase of migraine-what can we learn from it? Headache May 55(5):609-620

18. Schulte LH, May A (2016) The migraine generator revisited: continuous scanning of the migraine cycle over 30 days and three spontaneous attacks. Brain Jul 139(Pt 7):1987-1993

19. Arkink EB, Schmitz N, Schoonman GG et al (2017) The anterior hypothalamus in cluster headache. Cephalalgia Oct 37(11):1039-1050

20. Ferraro S, Nigri A, Bruzzone MG et al (2019) Cluster headache: insights from resting-state functional magnetic resonance imaging. Neurol Sci May 40(Suppl 1):45-47 
21. Maniyar FH, Sprenger T, Monteith T, Schankin C, Goadsby PJ (2014) Brain activations in the premonitory phase of nitroglycerin-triggered migraine attacks. Brain Jan 137(Pt 1):232-241

22. Malo-Urries M, Hidalgo-Garcia C, Estebanez-de-Miguel E, Tricas-Moreno JM, Santos-Lasaosa S, Jahanshahi M (2018) Sensory function in cluster headache: an observational study comparing the symptomatic and asymptomatic sides. Neuropsychiatr Dis Treat 14:3363-3371

23. Marmura MJ, Pello SJ, Young WB (2010) Interictal pain in cluster headache. Cephalalgia Dec 30(12):1531-1534

24. Pedersen AS, Snoer A, Barloese M, Petersen A, Jensen RH (2021) Prevalence of pre-cluster symptoms in episodic cluster headache: Is it possible to predict an upcoming bout? Cephalalgia Jan 20:333102421989255

25. Hoffmann J, Baca SM, Akerman S (2019) Neurovascular mechanisms of migraine and cluster headache. J Cereb Blood Flow Metab Apr 39(4):573-594

26. Cittadini E, May A, Straube A, Evers S, Bussone G, Goadsby PJ (2006) Effectiveness of intranasal zolmitriptan in acute cluster headache: a randomized, placebo-controlled, double-blind crossover study. Arch Neuro/ Nov 63(11):1537-1542

27. Dixon R, Warrander A (1997) The clinical pharmacokinetics of zolmitriptan. Cephalalgia Oct 17(Suppl 18):15-20

28. Bahra A, Gawel MJ, Hardebo JE, Millson D, Breen SA, Goadsby PJ (2000) Oral zolmitriptan is effective in the acute treatment of cluster headache. Neurology May 9(9):1832-1839 54(

29. Maas HJ, Spruit MA, Danhof M, Della Pasqua OE (2008) Relevance of absorption rate and lag time to the onset of action in migraine. Clin Pharmacokinet 47(2):139-146

\section{Figures}




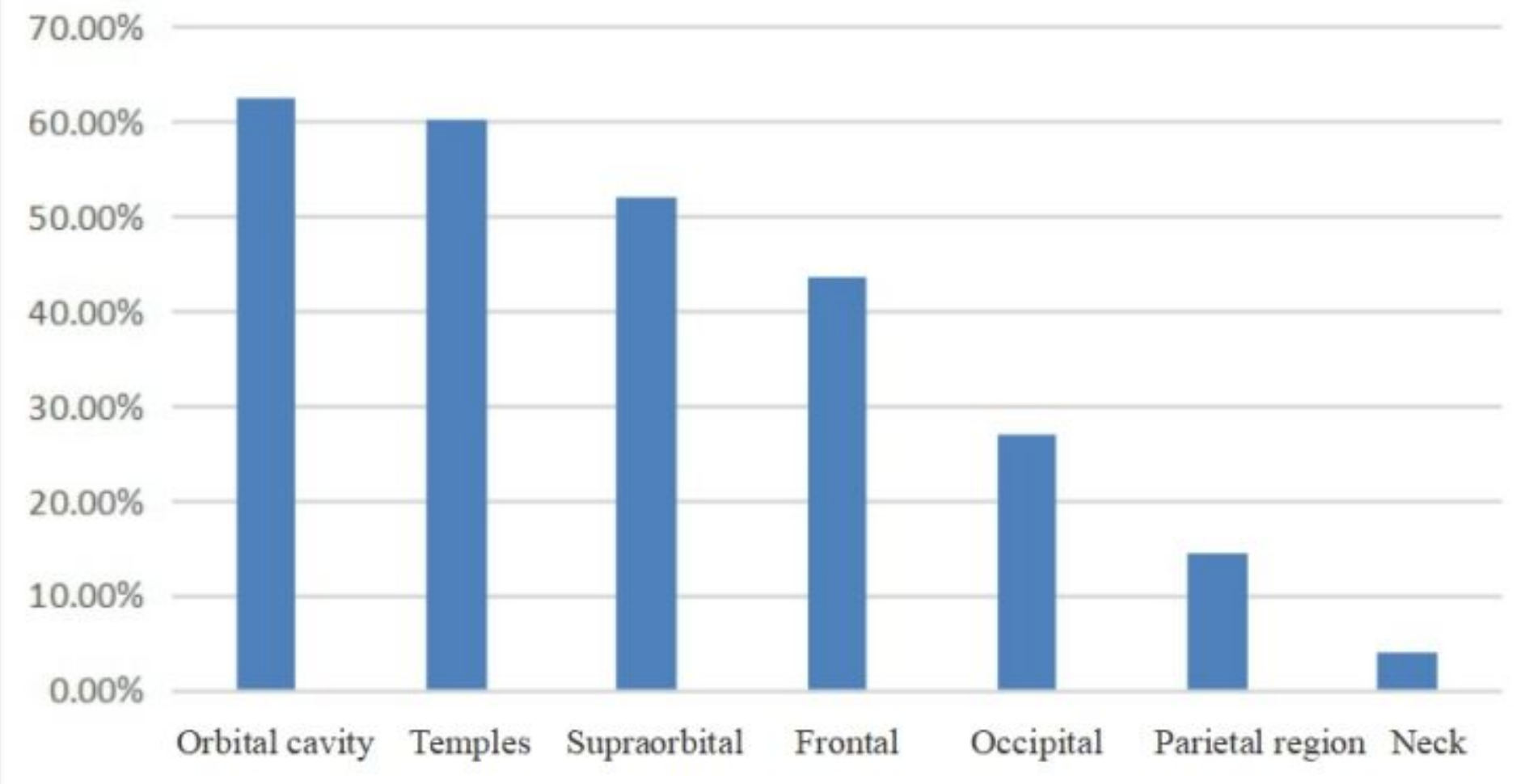

\section{Figure 1}

The locations of headache attack were multiple and the proportions were orbital $62.5 \%$, temporal $60.4 \%$, supraorbital $52.1 \%$, frontal $43.8 \%$, occipital $27.1 \%$, parietal region $14.6 \%$ and neck $4.2 \%$ 


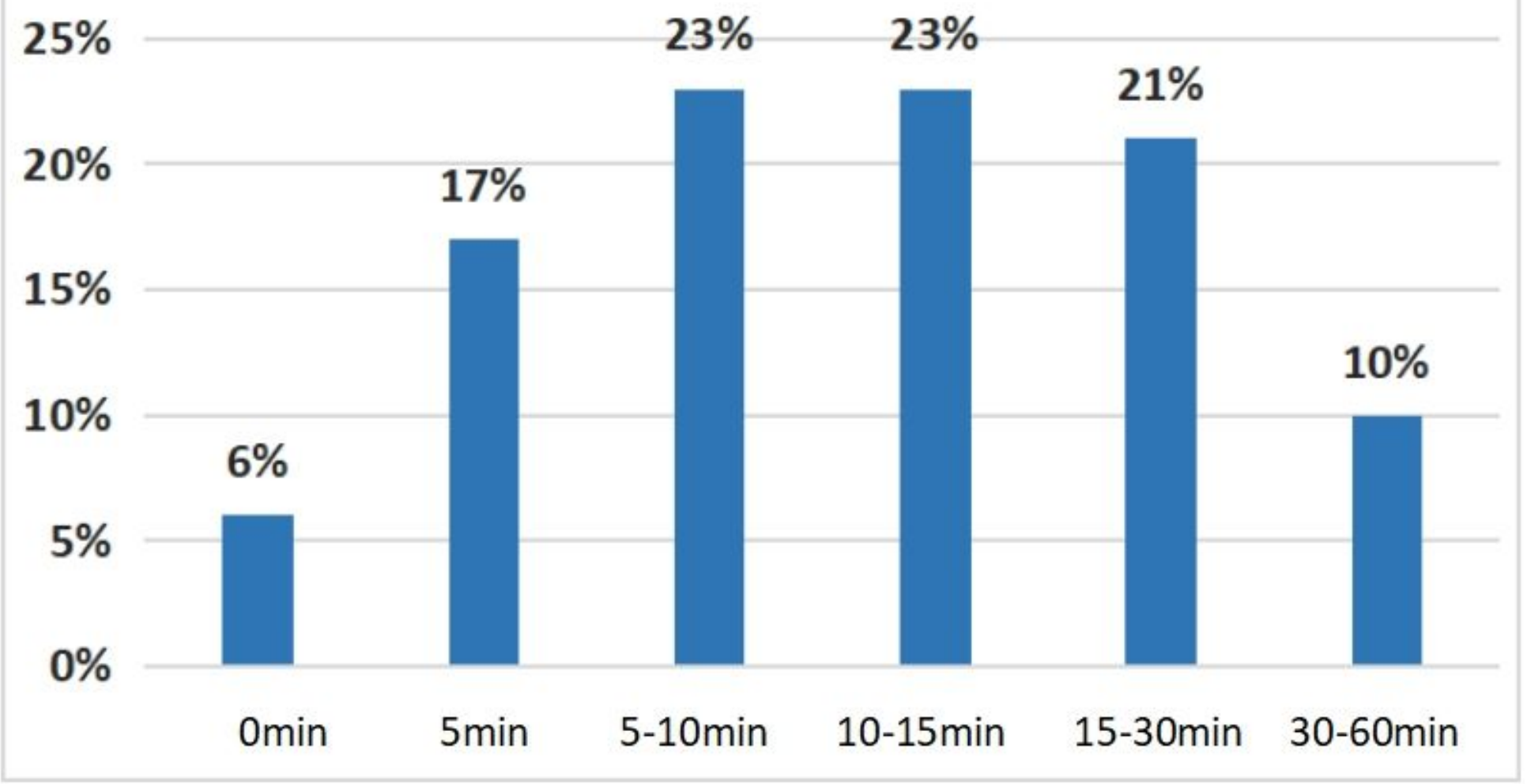

\section{Figure 2}

Time distribution of preictal phase $₫ 45$ had preictal phase, 3 patients reported no obvious preictal phase,8 patients with the duration of 5 minutes, 11 patients with 5 to 10 minutes, 11 with 10 to 15 minutes, 10 with 15 to 30 minutes, 5 with $30-60$ minutes. 


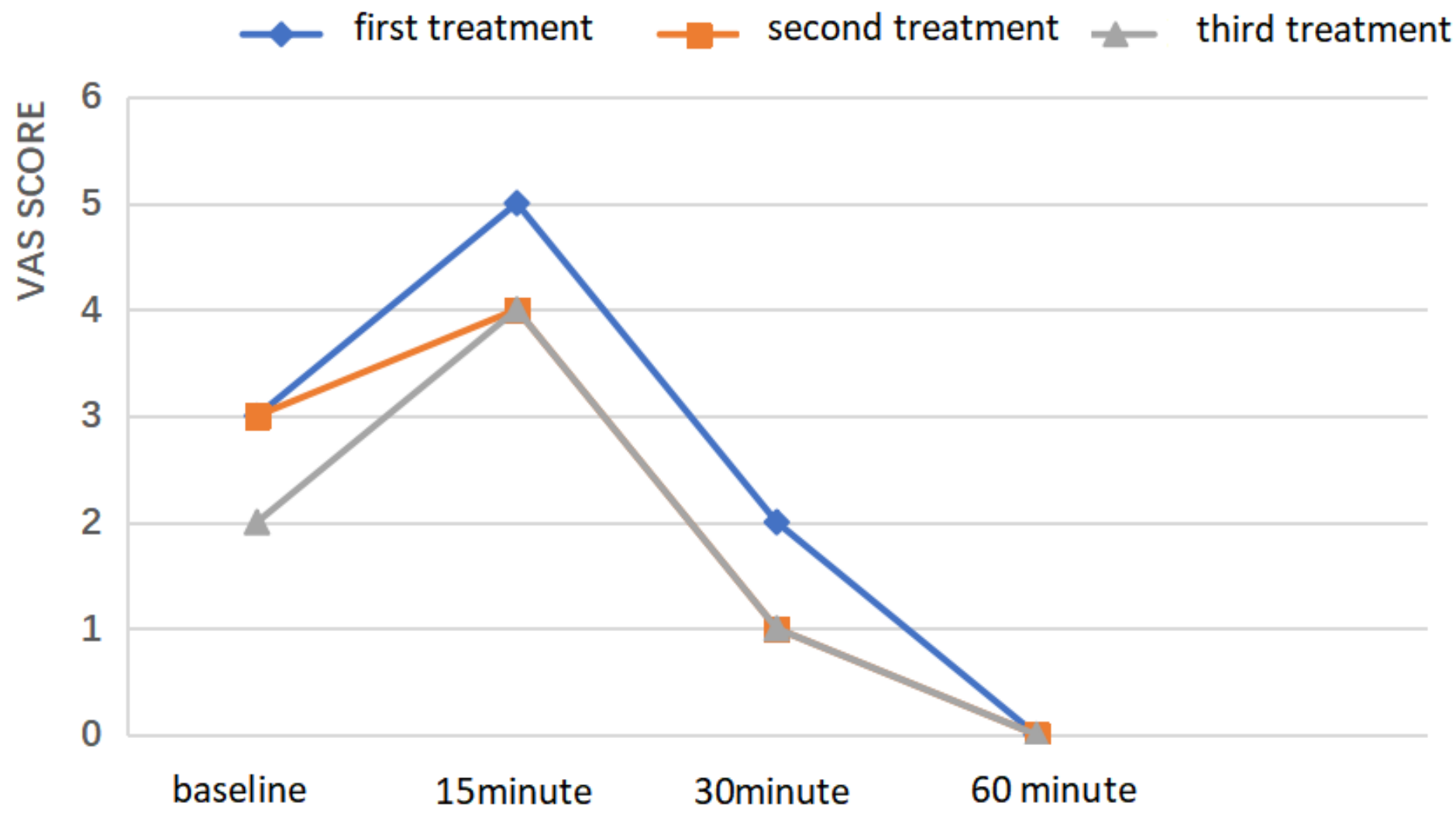

Figure 3

VAS score at different time points after three times preictal treatments 